\title{
Correction to: The role of osteocalcin in mechanism of steroid induced diabetes mellitus
}

\author{
Holla Avinash $^{1}$ (D) $\cdot$ Vidyasagar Sudha $^{1} \cdot$ Bairy Laxminarayan $^{2} \cdot$ B. Nandakrishna ${ }^{1} \cdot$ B. A. Shastry ${ }^{1} \cdot$ Kamath Asha $^{3}$. \\ Manjunath Hande ${ }^{1}$. Adiga Shalini ${ }^{4}$
}

Published online: 23 March 2020

(C) Research Society for Study of Diabetes in India 2020

\section{Correction to: International Journal of Diabetes in Developing Countries https://doi.org/10.1007/s13410-019-00791-6}

The manuscript used in the original version of this article was outdated. In this connection, the following errors were encountered.

1. Incorrect article title

2. Missing author Adiga Shalini

Because of this, the publisher regrets the error.

The original article has been corrected.

The online version of the original article can be found at https://doi.org/ 10.1007/s13410-019-00791-6

Vidyasagar Sudha

vsagar33@yahoo.com

1 Department of Medicine, Kasturba Medical College, Manipal, Academy of Higher Education, Udupi District,

Manipal, Karnataka 576104, India

2 Pharmacology RAK College of Medical Science, Ras Al, Khaimah, UAE

3 Department of Statistics, Prasanna School at Public Health, Manipal, Academy of Higher Education, Manipal, India

4 Department of Pharmacology, Kasturba Medical College, Manipal Academy of Higher Education, Udupi District,

Manipal, Karnataka 576104, India 